\title{
Orbital Magnetic Moment of Magnons
}

\author{
Robin R. Neumann, ${ }^{1}$ Alexander Mook, ${ }^{1,2}$ Jürgen Henk, ${ }^{1}$ and Ingrid Mertig ${ }^{1}$ \\ ${ }^{1}$ Institut für Physik, Martin-Luther-Universität Halle-Wittenberg, D-06099 Halle (Saale), Germany \\ ${ }^{2}$ Department of Physics, University of Basel, Klingelbergstrasse 82, CH-4056 Basel, Switzerland
}

(Dated: June 22, 2020)

\begin{abstract}
In experiments and applications usually the spin magnetic moment of magnons is considered. In this Paper we identify an additional degree of freedom of magnons: an orbital magnetic moment brought about by spin-orbit coupling. Our microscopic theory uncovers that spin magnetization $\boldsymbol{M}^{\mathrm{S}}$ and orbital magnetization $\boldsymbol{M}^{\mathrm{O}}$ are independent quantities. They are not necessarily collinear; thus, even when the total spin moment is compensated due to antiferromagnetism $\left(\boldsymbol{M}^{\mathrm{S}}=\mathbf{0}\right), \boldsymbol{M}^{\mathrm{O}}$ may be nonzero. This scenario of orbital weak ferromagnetism is realized in paradigmatic kagome antiferromagnets with Dzyaloshinskii-Moriya interaction. We demonstrate that magnets exhibiting a magnonic orbital moment are omnipresent and propose transport experiments for probing it.
\end{abstract}

Introduction. Textbooks on magnetism introduce spin waves as collective excitations of a magnetically ordered ground state, as epitomized by ferromagnets (Ref. 1 among others). The quanta of spin waves - the magnons - are typically viewed as local deviations from the ordered state [2,3]. Within this picture, it appears natural that the magnetic moment carried by magnons has only spatial components that are offered by the ground-state spin texture, because the latter defines the directions relative to which a deviation can occur [4]. This implies in particular that collinear magnets feature only magnons whose magnetic moment is along the collinear axis. Likewise the magnetic moments of magnons of coplanar magnets lie within that plane. This reasoning is widely accepted and adopted for a plethora of transport phenomena that involve the magnon magnetic moment, such as the spin Seebeck [5], spin Nernst [6-14], and magnon Edelstein effect $[15,16]$ in ferromagnets [17] and in both collinear [18-20] and noncollinear [13, 14, 21-23] antiferromagnets.

In this Paper, we challenge this paradigm by revealing an additional magnonic degree of freedom: their orbital magnetic moment. Overall, the magnetic moment

$$
\boldsymbol{\mu}_{n, \boldsymbol{k}}=-\frac{\partial \varepsilon_{n, \boldsymbol{k}}}{\partial \boldsymbol{B}}=\boldsymbol{\mu}_{n, \boldsymbol{k}}^{\mathrm{S}}+\boldsymbol{\mu}_{n, \boldsymbol{k}}^{\mathrm{O}}
$$

of a magnon in band $n$ and with momentum $\hbar \boldsymbol{k}$ decomposes into two contributions. These are derived from the explicit and implicit dependence of the magnon energy $\varepsilon_{n, k}$ with respect to the magnetic field $\boldsymbol{B}$. The first contribution,

$$
\mu_{n, k}^{S} \propto-s_{n, k},
$$

is the spin magnetic moment (SMM) which is proportional to the magnon spin $s_{n, k}[4,16]$. As mentioned above, this is the contribution conventionally referred to as the magnetic moment of magnons. The second contribution $\mu_{n, k}^{\mathrm{O}}-$ the orbital magnetic moment (OMM) - captures the difference of Eqs. (1) and (2) and is the main object of interest in this Paper. It is associated with an implicit dependence of $\varepsilon_{n, \boldsymbol{k}}$ on $\boldsymbol{B}$, which arises from the field-dependent relative orientation of the magnetic texture to the structural lattice [24] and, hence, requires spin-orbit coupling (SOC). The SMM and the OMM result in macroscopic spin and orbital magnetizations, $\boldsymbol{M}^{\mathrm{S}}$ and $\boldsymbol{M}^{\mathrm{O}}$, respectively. These independent quantities can be disentangled clearly in the situation of magnonic orbital weak ferromagnetism, in which $\boldsymbol{M}^{\mathrm{S}}=\mathbf{0}$ but $\boldsymbol{M}^{\mathrm{O}} \neq \mathbf{0}$. Importantly, even if $\boldsymbol{M}^{\mathrm{O}}=\mathbf{0}$ in equilibrium, the OMM may be addressed by an orbital Nernst effect of magnons in nonequilibrium. As a consequence, the complete set of magnonic degrees of freedom may be utilized for insulator spintronics.

Identification of the orbital magnetic moment. We start with a generic spin Hamiltonian $\hat{H}=\hat{H}_{\text {spin }}+\hat{H}_{\text {Zee }} ; \hat{H}_{\text {spin }}$ and $\hat{H}_{\text {Zee }}=\hbar^{-1} \mu_{\mathrm{B}} \sum_{i} \boldsymbol{B} \cdot \boldsymbol{g}_{i} \hat{\boldsymbol{S}}_{i}$ describe the spin-spin interactions (where the magnetic field $\boldsymbol{B}$ does not enter) and the coupling to the magnetic field (Zeeman term; $\hbar$ reduced Planck constant, $\mu_{\mathrm{B}}$ Bohr's magneton), respectively. $\boldsymbol{g}_{i}$ is the g-tensor of the spin operator $\hat{\boldsymbol{S}}_{i}$ at site $i$. Assuming an ordered ground state with $N$ spins per magnetic unit cell pointing along $\hat{z}_{n}(n=$ $1, \ldots, N)$, we perform a truncated Holstein-Primakoff (HP) transformation [25] from spin operators to bosonic operators $\hat{a}_{i}^{(\dagger)}$, yielding $\hat{H} \approx E_{0}+\hat{H}_{2}$. Here, $E_{0}$ is the classical ground state energy and $\hat{H}_{2}$ describes noninteracting magnons. After a transformation to magnonic normal modes $\hat{\alpha}_{n, \boldsymbol{k}}^{(\dagger)}$ in reciprocal space, we obtain $\hat{H} \approx E_{0}+\Delta E_{0}+\sum_{k} \sum_{n=1}^{N} \varepsilon_{n, k} \hat{\alpha}_{n, k}^{\dagger} \hat{\alpha}_{n, \boldsymbol{k}}$. The harmonic zero-point quantum fluctuations, $\Delta E_{0}=\Delta E_{0}^{(1)}+$ $\Delta E_{0}^{(2)}$, with $\Delta E_{0}^{(1)}=-\frac{1}{4} \sum_{\boldsymbol{k}} \operatorname{Tr} \boldsymbol{H}_{\boldsymbol{k}}$, and $\Delta E_{0}^{(2)}=\frac{1}{2} \sum_{\boldsymbol{k}} \sum_{n=1}^{N} \varepsilon_{n, \boldsymbol{k}}$, provide a correction to $E_{0} ; \boldsymbol{H}_{\boldsymbol{k}}$ is the Hamilton matrix. See Supplementary Material (SM) [26, Sec. I] for details. 
When considering effective spin Hamiltonians, usually the spin magnetization (SM [26, Sec. II])

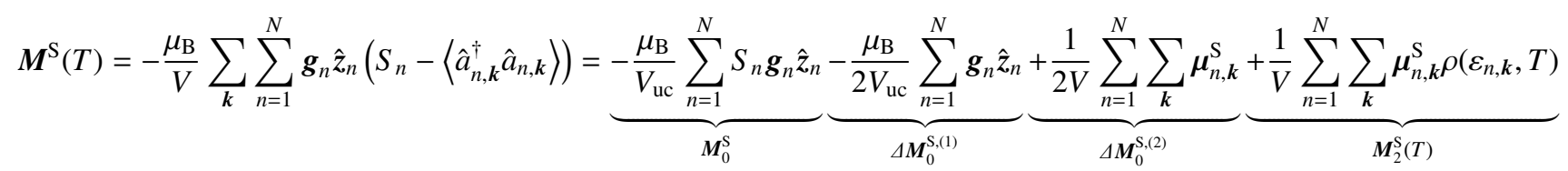

is addressed ( $V$ sample volume, $V_{\mathrm{uc}}$ volume of a unit cell, $\langle\cdot\rangle$ thermodynamic average). $S_{n}$ and $\boldsymbol{g}_{n}$ are the length and the $g$-tensor of the $n$th spin in the unit cell, respectively. Although $\boldsymbol{g}_{n}$ already incorporates SOC, we denote $\boldsymbol{M}^{\mathrm{S}}$ a "spin" magnetization, because the set $\left\{g_{n}\right\}$ merely transforms the directions $\hat{\boldsymbol{z}}_{n}$. The above sum is decomposed into the classical ground state spin magnetization $\boldsymbol{M}_{0}^{\mathrm{S}}$, its quantum corrections $\Delta \boldsymbol{M}_{0}^{\mathrm{S}}=\Delta \boldsymbol{M}_{0}^{\mathrm{S},(1)}+\Delta \boldsymbol{M}_{0}^{\mathrm{S},(2)}$, and into $\boldsymbol{M}_{2}^{\mathrm{S}}(T)$ which is due to the thermal population of magnons $\left[\rho\left(\varepsilon_{n k}, T\right)=\left(\mathrm{e}^{\beta \varepsilon_{n k}}-1\right)^{-1}\right.$ Bose-Einstein distribution function at temperature $\left.T=\left(k_{\mathrm{B}} \beta\right)^{-1}\right]$. Eventually, $\boldsymbol{\mu}_{n, \boldsymbol{k}}^{\mathrm{S}}$ is the $\mathrm{SMM}$ of magnons in band $n$ with momentum $\boldsymbol{k}$ (SM [26, Sec. II]).

$\boldsymbol{M}^{\mathrm{S}}(T)$ does not coincide with the thermodynamical definition of magnetization (SM [26, Sec. III])

$$
\boldsymbol{M}(T)=-\frac{1}{V} \frac{\partial \Omega}{\partial \boldsymbol{B}}=\underbrace{-\frac{1}{V} \frac{\partial E_{0}}{\partial \boldsymbol{B}}}_{\boldsymbol{M}_{0}} \underbrace{+\frac{1}{4 V} \sum_{\boldsymbol{k}} \frac{\partial \operatorname{Tr} \boldsymbol{H}_{\boldsymbol{k}}}{\partial \boldsymbol{B}}}_{\Delta \boldsymbol{M}_{0}^{(1)}=-\frac{1}{V} \frac{\partial \Delta E_{0}^{(1)}}{\partial \boldsymbol{B}}}+\underbrace{\frac{1}{2 V} \sum_{\boldsymbol{k}} \sum_{n=1}^{N} \boldsymbol{\mu}_{n, \boldsymbol{k}}}_{\Delta \boldsymbol{M}_{0}^{(2)}=-\frac{1}{V} \frac{\partial \Delta E_{0}^{(2)}}{\partial \boldsymbol{B}}} \underbrace{+\frac{1}{V} \sum_{n=1}^{N} \sum_{\boldsymbol{k}} \boldsymbol{\mu}_{n, \boldsymbol{k}} \rho\left(\varepsilon_{n, \boldsymbol{k}}, T\right)}_{\boldsymbol{M}_{2}(T)}
$$

( $\Omega$ grand potential). $\boldsymbol{\mu}_{n, \boldsymbol{k}}$ is the full magnonic magnetic moment defined in Eq. (1). The constituents of $\boldsymbol{M}$ are defined in analogy to those of $\boldsymbol{M}^{\mathrm{S}}$.

To verify briefly that $\boldsymbol{M}^{\mathrm{S}} \neq \boldsymbol{M}$ replace the g-tensor by a scalar $\left(\boldsymbol{g}_{n} \rightarrow g_{n}\right) . \boldsymbol{M}^{\mathrm{S}}$ is then restricted to those spatial components offered by the $\hat{z}_{n}$ 's; however, $\boldsymbol{M}$ and $\boldsymbol{\mu}_{n, \boldsymbol{k}}$ are not, because the $\hat{\boldsymbol{z}}_{n}$ 's themselves depend on $\boldsymbol{B}$. Thus, even if all $\hat{\boldsymbol{z}}_{n}$ 's are collinear (or coplanar), $\boldsymbol{\mu}_{n k}$ may have an orthogonal component whose integral is nonzero; hence, $\boldsymbol{M} \nVdash \boldsymbol{M}^{\mathrm{S}}$.

The observation $\boldsymbol{M}_{0}=\boldsymbol{M}_{0}^{\mathrm{S}}(\mathrm{SM}[26$, Sec. IV] $)$ allows to trace the difference of $\boldsymbol{M}$ and $\boldsymbol{M}^{\mathrm{S}}$ back to the difference between $\boldsymbol{\mu}_{n, \boldsymbol{k}}$ and $\boldsymbol{\mu}_{n, \boldsymbol{k}}^{\mathrm{S}}$. More precisely, one obtains $\boldsymbol{\mu}_{n, \boldsymbol{k}}=\boldsymbol{\mu}_{n, \boldsymbol{k}}^{\mathrm{S}}+\boldsymbol{\mu}_{n, \boldsymbol{k}}^{\mathrm{O}}$, in which the SMM is derived from the explicit $\boldsymbol{B}$ dependence of the Zeeman energy and the OMM (SM [26, Sec. V])

$$
\boldsymbol{\mu}_{n, \boldsymbol{k}}^{\mathrm{O}}=-\sum_{m=1}^{N} \sum_{\alpha=x, y, z} \frac{\partial \varepsilon_{n, \boldsymbol{k}}}{\partial \hat{\boldsymbol{\alpha}}_{m}} \cdot \frac{\partial \hat{\boldsymbol{\alpha}}_{m}}{\partial \boldsymbol{B}}
$$

from the implicit $\boldsymbol{B}$ dependence of the local coordinate system $\left\{\hat{\boldsymbol{x}}_{n}, \hat{\boldsymbol{y}}_{n}, \hat{\boldsymbol{z}}_{n}\right\}$ [27]. Such a dependence has to result from SOC (or SOC-like interactions) which couples spins and lattice and therefore motivates the term "orbital" moment. The orbital magnetization

$$
\boldsymbol{M}^{\mathrm{O}}(T)=\Delta \boldsymbol{M}_{0}^{\mathrm{O},(1)}+\underbrace{\frac{1}{2 V} \sum_{\boldsymbol{k}} \sum_{n=1}^{N} \boldsymbol{\mu}_{n, \boldsymbol{k}}^{\mathrm{O}}}_{\Delta \boldsymbol{M}_{0}^{\mathrm{O}(2)}} \underbrace{\frac{1}{V} \sum_{n=1}^{N} \sum_{\boldsymbol{k}} \boldsymbol{\mu}_{n, \boldsymbol{k}}^{\mathrm{O}} \rho\left(\varepsilon_{n \boldsymbol{k}}, T\right)}_{\boldsymbol{M}_{2}^{\mathrm{O}}(T)}
$$

is absent in the classical ground state, since it is exclusively due to quantum $\left(\Delta \boldsymbol{M}_{0}^{\mathrm{O},(1)}+\Delta \boldsymbol{M}_{0}^{\mathrm{O},(2)}\right)$ and thermal fluctuations $\left(\boldsymbol{M}_{2}^{\mathrm{O}}(T)\right)$.
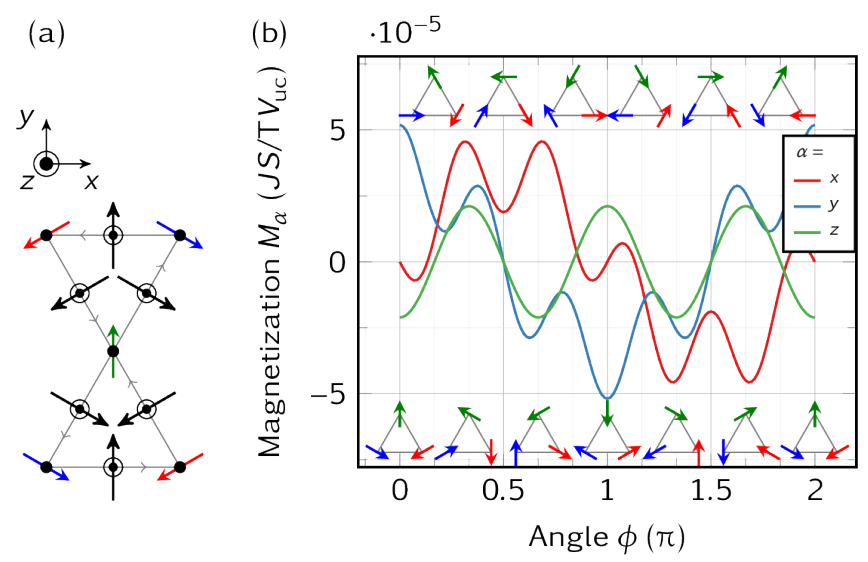

FIG. 1. Weak ferromagnetism due to quantum fluctuations in the NVC phase on the kagome lattice. (a) Structural lattice with $\hat{z}_{n}(n=1,2,3)$ indicated by colored arrows and DMI vectors by black arrows. (b) Magnetization components $M_{\alpha}(\alpha=x, y, z)$ at zero temperature in dependence on $\phi$. Since $|\boldsymbol{M}| \neq 0$, quantum weak ferromagnetism is omnipresent; the out-of-plane magnetization $M_{z}=M_{z}^{\mathrm{O}}$ is attributed to an orbital moment. Parameters read $J=3.18 \mathrm{meV}, S=5 / 2$, $D_{z}=0.062 \mathrm{~J}, D_{\|}=1 \mathrm{meV}$.

In what follows, we assume scalar $g$-factors and include SOC exclusively via spin-spin interactions.

Orbital magnetic moments in equilibrium. First, we demonstrate how OMM can be probed in equilibrium as a contribution to weak ferromagnetism. This phenomenon is usually described at the level of classically antiferromagnetic spin textures that exhibit a small canting, e. g., due to DzyaloshinskiiMoryia interaction (DMI) $[28,29]\left(\boldsymbol{M}_{0}^{\mathrm{S}} \neq \mathbf{0}\right)$. Here, we predict 
TABLE I. Magnetic point group and symmetry-imposed shape of $\boldsymbol{M}$ for NVC phases with $\phi=0$ and $\phi=\pi / 2$.

\begin{tabular}{cccc}
\hline Angle $\phi$ & 0 & $\pi / 2$ \\
\hline Magnetic point group & $2^{\prime} / m^{\prime}$ & $2 / m$ \\
Compatible magnetization & $\left(\begin{array}{lllll}0 & M_{y} & M_{z}\end{array}\right)\left(\begin{array}{lll}M_{x} & 0 & 0\end{array}\right)$ \\
\hline
\end{tabular}

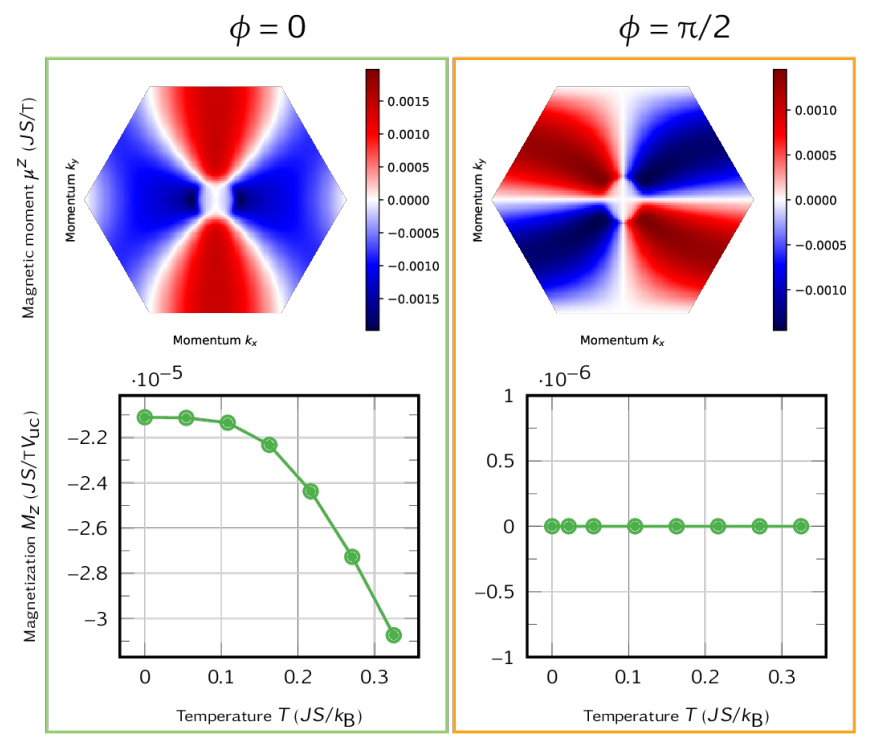

FIG. 2. Top: momentum-dependent OMM $\mu_{1, k, z}$ of the lowest magnon band $(n=1)$ in the Brillouin zone of the kagome antiferromagnet in the NVC phase for $\phi=0$ (left) and $\phi=\pi / 2$ (right). Bottom: temperature dependence of the orbital magnetization $M_{z}$. Parameters as in Fig. 1.

pure orbital weak ferromagnetism: $\boldsymbol{M}_{0}^{\mathrm{S}}=\mathbf{0}$ but $\boldsymbol{M}^{\mathrm{O}} \neq \mathbf{0}$. A system of choice is a kagome antiferromagnet [Fig. 1(a)] with the spin Hamiltonian

$$
\hat{H}=\frac{1}{2 \hbar^{2}} \sum_{\langle i j\rangle}\left(-J \hat{\boldsymbol{S}}_{i} \cdot \hat{\boldsymbol{S}}_{j}+\boldsymbol{D}_{i j} \cdot \hat{\boldsymbol{S}}_{i} \times \hat{\boldsymbol{S}}_{j}\right)+\frac{g \mu_{\mathrm{B}}}{\hbar} \boldsymbol{B} \cdot \sum_{i} \hat{\boldsymbol{S}}_{i}
$$

whose classical phase diagram was derived in Ref. 30. Each spin interacts with its four neighbours via antiferromagnetic exchange $J<0$ and SOC-induced DMI. The DMI vectors $\boldsymbol{D}_{i j}$ are orthogonal to the respective bond [black arrows in Fig 1(a)] and have both an in-plane $\left(D_{\|}\right)$and an out-of-plane components $\left(D_{z}\right)$. For $D_{z}>0$ and $\left|D_{\|}\right|$below a critical value, the classical magnetic ground state is an antiferromagnetic coplanar texture with negative vector chirality (NVC) [30] [colored arrows in Fig. 1(a)]. The classical spin magnetization vanishes $\left(\boldsymbol{M}_{0}^{\mathrm{S}}=\mathbf{0}\right)$. For $E_{0}$ exhibits an accidental degeneracy under global in-plane rotation of all spins, we perform an order-by-disorder study with respect to the rotation angle $\phi$ [insets in Fig. 1(b)]. Both quantum and thermal fluctuations select the $\phi=0$ texture [Fig. 1(a)] and its $\pi / 3$ rotations over any other rotated texture (SM [26, Sec. VI]). Nonetheless, we proceed with studying all textures.

For the discussion we single out the phases for $\phi=0$ with magnetic point group $2^{\prime} / m^{\prime}$ (the prime indicates additional time reversal) and $\phi=\pi / 2$ with $2 / m$ [31]. In both cases the two-fold rotation axis is along the $x$ direction and the mirror plane coincides with the $y z$ plane. Both groups are compatible with ferromagnetism (Tab. I). Besides an in-plane magnetization, the $\phi=0$ phase is also compatible with a nonzero $M_{z}$. Since $\hat{z}_{n, z}=0$ by construction, any nonzero $M_{z}=M_{z}^{\mathrm{O}}$ must be attributed to an orbital moment.

This symmetry analysis is fully confirmed by the magnetization calculated from Eq. (4) [Fig. 1(b)]. Although $\boldsymbol{M}_{0}(\phi)=\boldsymbol{M}_{0}^{\mathrm{S}}(\phi)=\mathbf{0}$ for all $\phi$, the quantum-corrected magnetization is never compensated: $\left|\Delta \boldsymbol{M}_{0}(\phi)\right| \neq 0$. Hence, the quantum fluctuations cause the weak ferromagnetism, of both spin and orbital origin for $M_{x}$ and $M_{y}$ but of pure orbital origin for $M_{z}$. This finding complements classical analyses of kagome antiferromagnets [30] and shows that even the NVC phase exhibits weak ferromagnetism without the need of higher-order anisotropies beyond DMI [32]. It is also a counterexample to the common belief that quantum fluctuations only reduce the magnitude of the ordered moment.

The microscopic origin of $M_{z} \neq 0$ can be studied on the basis of the OMM $\mu_{1, k, z}=\mu_{1, k, z}^{\mathrm{O}}$ of the lowest magnon band $(n=1)$ for both phases (top row of Fig. 2; recall $\mu_{1, k, z}^{\mathrm{S}}=0$ ). Already an "ocular integration" over the Brillouin zone reveals that $M_{z}(T)=M_{z}^{\mathrm{O}}(T)$ from Eq. (6) must be either nonzero $(\phi=0)$ or zero $(\phi=\pi / 2)$, an observation confirmed by numerical integration (bottom row of Fig. 2). For the $\phi=0$ phase $\left|M_{z}(T)\right|$ increases in absolute value with temperature, showing that thermal fluctuations enhance the quantum mechanical weak moment (the $T$ dependence of $M_{x}$ and $M_{y}$ is detailed in SM $[26, \mathrm{Sec} . \mathrm{VI}])$.

That SOC is causing the orbital moment is supported by noting that $\mu_{n, k, z}, M_{z}(T) \rightarrow 0$ as $D_{\|} \rightarrow 0$ (not shown). If $D_{\|}=0$ the kagome plane is an $m^{\prime}$ plane, which renders $M_{z}$ zero by symmetry. Hence, in the absence of SOC-induced spin-spin interactions, the orbital magnetization vanishes.

Orbital magnetic moments in nonequilibrium. Having established signatures of OMMs at equilibrium, we now focus on nonequilibrium and consider as an example transport of magnetic moment - rather than spin - in the pyrochlore ferromagnet $\mathrm{Lu}_{2} \mathrm{~V}_{2} \mathrm{O}_{7}$. The spin Hamiltonian [33]

$$
\hat{H}=\frac{1}{2 \hbar^{2}} \sum_{\langle i j\rangle}\left(-J \hat{\boldsymbol{S}}_{i} \cdot \hat{\boldsymbol{S}}_{j}+\boldsymbol{D}_{i j} \cdot \hat{\boldsymbol{S}}_{i} \times \hat{\boldsymbol{S}}_{j}\right)+\frac{g \mu_{\mathrm{B}}}{\hbar} \boldsymbol{B} \cdot \sum_{i} \hat{\boldsymbol{S}}_{i},
$$

includes DMI vectors $\boldsymbol{D}_{i j}=D \hat{\boldsymbol{n}}_{i j} \times \hat{\boldsymbol{e}}_{i j}$ that are perpendicular to both the bonds $\hat{\boldsymbol{e}}_{i j}$ and the normal $\hat{\boldsymbol{n}}_{i j}$ of the cube that surrounds that tetrahedron the bond belongs to [34]. For $J>0$, collinear ferromagnetism is found, $\hat{z}_{n}=-\hat{\boldsymbol{b}}=-\boldsymbol{B} / \boldsymbol{B}(n=1, \ldots, 4)$, and quantum fluctuations are absent, $\Delta \boldsymbol{M}_{0}=\mathbf{0}$.

The application of a magnetic field $\boldsymbol{B}=\left(0,0, B_{z}\right)$ [35] results in $\mu_{n, \boldsymbol{k}, z}=\mu_{n, \boldsymbol{k}, z}^{\mathrm{S}}=g \mu_{\mathrm{B}}$ and $\mu_{n, \boldsymbol{k}, \alpha}=\mu_{n, \boldsymbol{k}, \alpha}^{\mathrm{O}}=O(D)$ for $\alpha=x, y$. Hence, the constant $z$ component of $\boldsymbol{\mu}_{n, \boldsymbol{k}}$ is a SMM. The $x$ and $y$ components are OMMs, however, which for positive (negative) $k_{z}$ resembles a sink-like (source-like) vector field, as depicted in Fig. 3. 

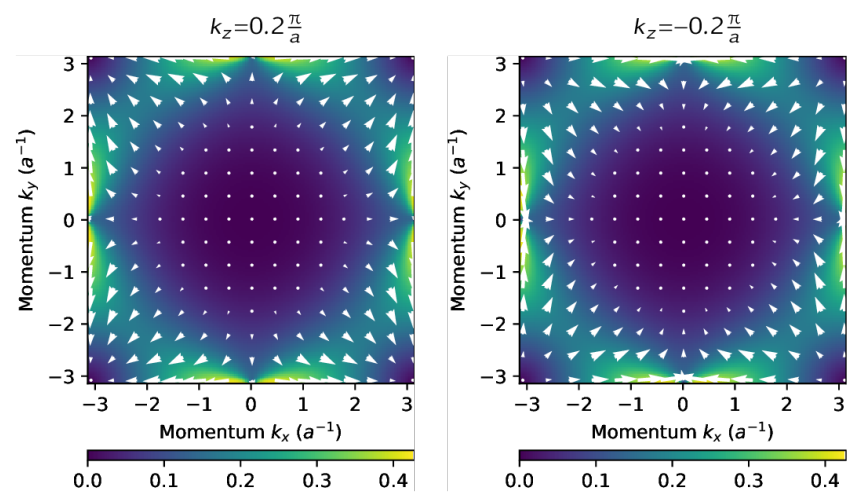

FIG. 3. Orbital magnetic moments $\mu_{k, x}$ and $\mu_{k, y}$ of the lowest magnon band of the pyrochlore ferromagnet $\mathrm{Lu}_{2} \mathrm{~V}_{2} \mathrm{O}_{7}$. The color scale represents $\sqrt{\mu_{\boldsymbol{k}, x}^{2}+\mu_{\boldsymbol{k}, y}^{2}}$ (in units of $\mu_{\mathrm{B}}$ ) in two selected $k_{x}-k_{y}$ planes: $k_{z}=0.2 \pi / a$ (left) and $k_{z}=-0.2 \pi / a$ (right); $a$ lattice constant.

TABLE II. Shape of response tensors $\gamma^{\gamma}(\gamma=x, y, z)$ for the magnetic point group $4 / \mathrm{mm}^{\prime} \mathrm{m}^{\prime}$. A subscript "e" ("o") indicates elements that are even (odd) under magnetization reversal.

\begin{tabular}{|c|c|c|c|c|c|c|c|c|}
\hline & $\Upsilon^{x}$ & & & $\Upsilon^{y}$ & & & $\gamma^{z}$ & \\
\hline 0 & 0 & $\Upsilon_{\mathrm{o}}$ & 0 & 0 & $\left.\Upsilon_{\mathrm{e}}\right)$ & $\tilde{\Upsilon}_{\mathrm{o}}$ & $\tilde{\Upsilon}_{\mathrm{e}}$ & 0 \\
\hline 0 & 0 & $-\Upsilon_{\mathrm{e}}$ & 0 & 0 & $\Upsilon_{\mathrm{o}}$ & $-\tilde{\Upsilon}_{\mathrm{e}}$ & $\tilde{\Upsilon}_{\mathrm{o}}$ & 0 \\
\hline$\left(\Upsilon_{\mathrm{o}}^{\prime}\right.$ & $-\Upsilon_{\mathrm{e}}^{\prime}$ & 0 & $\left(\gamma_{\mathrm{e}}^{\prime}\right.$ & $\Upsilon_{\mathrm{o}}^{\prime}$ & 0 & 0 & 0 & $\tilde{\Upsilon}_{\mathrm{o}}^{\prime}$ \\
\hline
\end{tabular}

In equilibrium, the OMM integrates to zero, $\boldsymbol{M}_{2}^{\mathrm{O}}(T)=\mathbf{0}$. However, in nonequilibrium, it is transported in transverse direction to a temperature gradient $\nabla T$. In other words, this is a Nernst effect (NE) for magnetic moment rather than for spin. Its analysis focuses on the response tensor $\gamma^{\gamma}$ which relates the nonequilibrium current density of the magnetization with the temperature gradient: $\left\langle j_{\alpha}^{\gamma}\right\rangle=\Upsilon_{\alpha \beta}^{\gamma}\left(-\nabla_{\beta} T\right)$ with $\alpha, \beta, \gamma=x, y, z$.

Pyrochlore ferromagnets magnetized in $z$ direction belong to the magnetic point group $4 / \mathrm{mm}^{\prime} \mathrm{m}^{\prime}$, which dictates the shape of $\Upsilon^{\gamma}$ (Tab. II). Tensor elements that are even upon magnetization reversal (subscript "e") are associated with intrinsic contributions to the transport, whereas odd elements (subscript "o") are associated with extrinsic contributions [13, 37].

The elements $\tilde{\Upsilon}_{\mathrm{o}}$ and $\tilde{\Upsilon}_{\mathrm{o}}^{\prime}$ of $\Upsilon^{z}$ comprise a spin Seebeck effect, while $\tilde{\Upsilon}_{\mathrm{e}}$ indicates an anomalous spin Nernst effect (SNE) which is associated with spin-polarized transverse particle currents caused by the Berry curvature [12, 31, 33, 38-42].

Besides transport of the $z$-component, symmetry admits transport of $x$ - and $y$-components as well ( $\Upsilon^{x}$ and $\Upsilon^{y}$ in Tab. II). $\Upsilon_{\mathrm{e}}\left(\Upsilon_{\mathrm{e}}^{\prime}\right)$ comprises an anomalous SNE with mutual orthogonality of force, current, and moment directions, whereas $\Upsilon_{\mathrm{o}}\left(\Upsilon_{\mathrm{o}}^{\prime}\right)$ indicates a magnetic SNE [14]. Since the $x$ - and $y$-components are OMMs, the respective SNEs could be termed "magnonic orbital Nernst effects".

The above symmetry analysis suggests straightaway an experimental setup for probing OMMs. In a finite pyrochlore sample with $-\boldsymbol{\nabla} T\|\boldsymbol{M}\| \boldsymbol{z}$, OMM accumulates at the surfaces parallel to $\boldsymbol{M}$ ( $x z$ and $y z$ surfaces). The resulting surface- located nonequilibrium tilt on $\boldsymbol{M}$, conceivably measured by magnetooptical Kerr microscopy, would clearly indicate transport of magnonic orbital magnetization.

We support the above analysis by calculating numerically all 27 elements of $\Upsilon^{\gamma}$ within Kubo transport theory (Fig. 4; SM [26, Sec. VII]). Vanishing elements (marked by yellow background) agree with the zeroes in Tab. II; and so does the either intrinsic (blue, "e") or extrinsic (red, "o") character. Except for the diagonal elements of $\Upsilon^{z}$, all elements scale with the strength $D$ of the DMI, because DMI causes either a nonzero Berry curvature $\left(\tilde{\Upsilon}_{\mathrm{e}}\right)$ or OMMs $\left(\Upsilon_{\mathrm{e}}, \Upsilon_{\mathrm{e}}^{\prime}, \Upsilon_{\mathrm{o}}, \Upsilon_{\mathrm{o}}^{\prime}\right)$. With an orbital Nernst conductivity $\Upsilon_{x z}^{x} \approx-0.4 \mathrm{~mJ} /(\mathrm{TKms})$ at $T=20 \mathrm{~K}$ and $\nabla_{z} T=25 \mathrm{~K} / \mathrm{mm}$, we find $\left\langle j_{x}^{x}\right\rangle \approx 10 \mathrm{~J} /\left(\mathrm{Tm}^{2} \mathrm{~s}\right)$ (in units of spin, this corresponds to $\hbar\left\langle j_{x}^{x}\right\rangle / \mu_{\mathrm{B}} \sim 10^{-10} \mathrm{~J} / \mathrm{m}^{2}$ ).

Another class of magnets lends itself support for nontrivial magnonic OMMs: chiral magnets, like $\mathrm{Cu}_{2} \mathrm{OSeO}_{3}$, which hold a prominent place in skyrmion research [43]. Their DMIinduced magnonic OMM $\boldsymbol{\mu}_{\boldsymbol{k}}^{\mathrm{O}}$ is nonzero, but integrates to $\boldsymbol{M}^{\mathrm{O}}=$ 0 in equilibrium. Due to broken centrosymmetry, however, a magnon current caused by $-\boldsymbol{\nabla} T$ exerts a torque on $\boldsymbol{M}[8,44]$, an effect that can be explained as an orbital version of the magnon Edelstein effect proposed in Ref. 16; see SM [26, Sec. VIII].

Dipolar interactions couple spins to the lattice as well. A magnonic OMM - or better: dipolar magnetic moment - could be identified as follows. Magnons with $\boldsymbol{k} \nVdash \boldsymbol{M}$ carry nonzero $\boldsymbol{\mu}_{\boldsymbol{k}}^{\mathrm{O}} \perp \boldsymbol{M}$. Again, $\boldsymbol{M}^{\mathrm{O}}=\mathbf{0}$ in equilibrium, but a dipolar-driven "orbital" Nernst effect should show up for symmetry reasons, for example in yttrium iron garnet (YIG); see SM [26, Sec. IX].

Synopsis. We introduced the orbital magnetic moment of magnons and proposed two experimental signatures: (1) weak ferromagnetic orbital moment in equilibrium and (11) accumulation of orbital magnetic moment in nonequilibrium due to a magnonic orbital Nernst effect. Since the latter has the same symmetry as the spin Hall effect [45], it should occur in any magnet with large enough SOC or dipolar interactions. Hence, our results pave a way for an all-insulator magnonic spin-orbit torque.

Acknowledegments. This work is supported by CRC/TRR 227 of Deutsche Forschungsgemeinschaft (DFG).

Note added. The magnonic OMM defined as the difference between total moment and SMM applies to any spin Hamiltonian. For the Hamiltonians discussed in this work, it can be traced back to the dependence of the local coordinate axes on the magnetic field as written in Eq. 5. The "topological orbital moment" and the resulting orbital Nernst effect of magnons discussed in Ref. 46, both of which rely on a special type of spin interaction, namely three-spin ring exchange, is also captured by Eq. 1 and would appear as an additional contribution in Eq. 5. However, in the frame of this work, we confined ourselves to bilinear spin-spin interactions. 

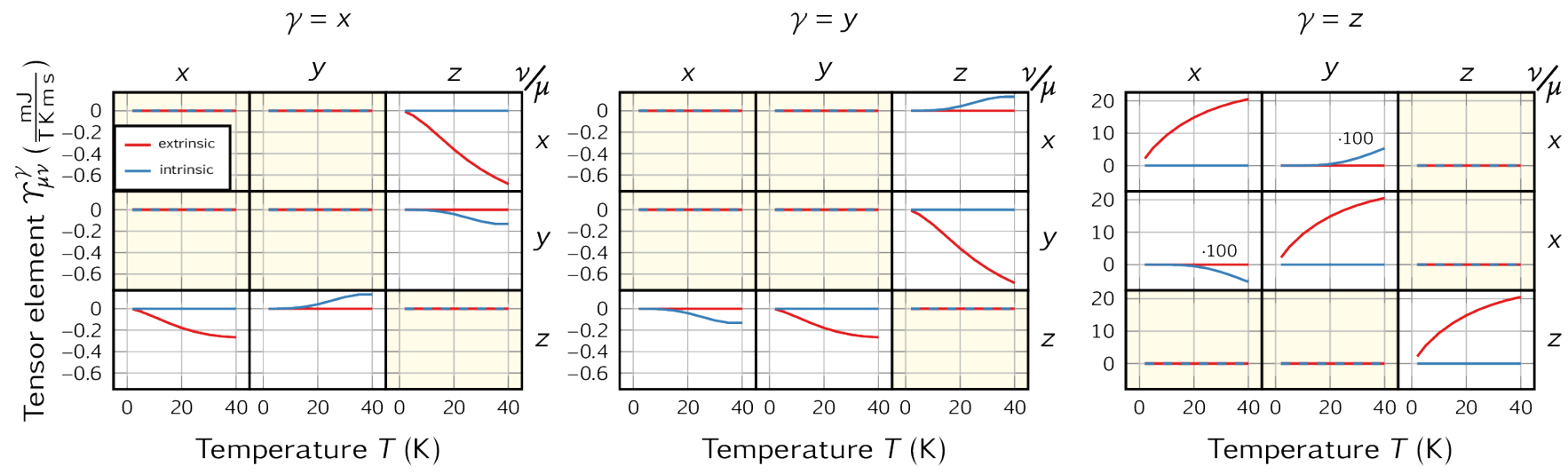

FIG. 4. Transport of magnetic moment in the pyrochlore ferromagnet $\mathrm{Lu}_{2} \mathrm{~V}_{2} \mathrm{O}_{7}$. The temperature dependence of all 27 elements of the response tensors $r^{\gamma}$ are depicted: $\gamma=x$ (left), $\gamma=y$ (center), and $\gamma=z$ (right). In each of the 3-by-3 subfigures, rows (columns) represent the current direction $\mu$ (direction $v$ of the temperature gradient). The yellow background highlights vanishing elements. For the extrinsic contributions (red lines), a transport relaxation time $\tau_{n k}=\hbar /\left(\alpha \varepsilon_{n k}\right)$ with $\alpha=0.05$ is assumed. Intrinsic contributions (blue lines) are calculated in the so-called clean limit. Parameters read $J=-7.99 \mathrm{meV}, S=1 / 2, D=0.5659 \mathrm{meV}, a=2.49 \AA[33,36]$, and $B_{z}=-0.69 \mathrm{~T}$. The ordering temperature of $\mathrm{Lu}_{2} \mathrm{~V}_{2} \mathrm{O}_{7}$ is $70 \mathrm{~K}[33]$.

[1] N.W. Ashcroft and N.D. Mermin, Solid State Physics (Saunders College, Philadelphia, 1976).

[2] F. Bloch, "Zur Theorie des Ferromagnetismus," Zeitschrift für Physik 61, 206-219 (1930).

[3] F. Bloch, "Zur Theorie des Austauschproblems und der Remanenzerscheinung der Ferromagnetika," Zeitschrift für Physik 74, 295-335 (1932).

[4] Nobuyuki Okuma, "Magnon spin-momentum locking: Various spin vortices and Dirac magnons in noncollinear antiferromagnets," Phys. Rev. Lett. 119, 107205 (2017).

[5] K. Uchida, J. Xiao, H. Adachi, J. Ohe, S. Takahashi, J. Ieda, T. Ota, Y. Kajiwara, H. Umezawa, H. Kawai, and et al., "Spin Seebeck insulator," Nature Materials 9, 894897 (2010).

[6] Jung Hoon Han and Hyunyong Lee, "Spin chirality and Hall-like transport phenomena of spin excitations," Journal of the Physical Society of Japan 86, 011007 (2017).

[7] Ran Cheng, Satoshi Okamoto, and Di Xiao, "Spin Nernst effect of magnons in collinear antiferromagnets," Phys. Rev. Lett. 117, 217202 (2016).

[8] Alexey A. Kovalev and Vladimir Zyuzin, "Spin torque and Nernst effects in Dzyaloshinskii-Moriya ferromagnets," Phys. Rev. B 93, 161106 (2016).

[9] Alexander Mook, Jürgen Henk, and Ingrid Mertig, "Spin dynamics simulations of topological magnon insulators: From transverse current correlation functions to the family of magnon Hall effects," Phys. Rev. B 94, 174444 (2016).

[10] Y. Shiomi, R. Takashima, and E. Saitoh, "Experimental evidence consistent with a magnon Nernst effect in the antiferromagnetic insulator $\mathrm{MnPS}_{3}$," Phys. Rev. B 96, 134425 (2017).

[11] X. S. Wang and X. R. Wang, "Anomalous magnon Nernst effect of topological magnonic materials," Journal of Physics D: Applied Physics 51, 194001 (2018).

[12] Alexander Mook, Börge Göbel, Jürgen Henk, and Ingrid Mertig, "Taking an electron-magnon duality shortcut from electron to magnon transport," Phys. Rev. B 97, 140401 (2018).

[13] Alexander Mook, Robin R. Neumann, Jürgen Henk, and Ingrid Mertig, "Spin Seebeck and spin Nernst effects of magnons in noncollinear antiferromagnetic insulators," Phys. Rev. B 100, 100401 (2019)

[14] Alexander Mook, Robin R. Neumann, Annika Johansson, Jürgen Henk, and Ingrid Mertig, "Origin of the magnetic spin Hall effect: Spin current vorticity in the Fermi sea," Phys. Rev. Research 2, 023065 (2020).

[15] Atsuo Shitade and Youichi Yanase, "Magnon gravitomagnetoelectric effect in noncentrosymmetric antiferromagnetic insulators," Phys. Rev. B 100, 224416 (2019).

[16] Bo Li, Alexander Mook, Aldo Raeliarijaona, and Alexey A. Kovalev, "Magnonic analog of the Edelstein effect in antiferromagnetic insulators," Phys. Rev. B 101, 024427 (2020).

[17] Joel Cramer, Er-Jia Guo, Stephan Geprägs, Andreas Kehlberger, Yurii P. Ivanov, Kathrin Ganzhorn, Francesco Della Coletta, Matthias Althammer, Hans Huebl, Rudolf Gross, Jürgen Kosel, Mathias Kläui, and Sebastian T. B. Goennenwein, "Magnon mode selective spin transport in compensated ferrimagnets," Nano Letters 17, 3334-3340 (2017).

[18] S. Seki, T. Ideue, M. Kubota, Y. Kozuka, R. Takagi, M. Nakamura, Y. Kaneko, M. Kawasaki, and Y. Tokura, "Thermal generation of spin current in an antiferromagnet," Phys. Rev. Lett. 115, 266601 (2015).

[19] Stephen M. Wu, Wei Zhang, Amit KC, Pavel Borisov, John E. Pearson, J. Samuel Jiang, David Lederman, Axel Hoffmann, and Anand Bhattacharya, "Antiferromagnetic spin Seebeck effect," Phys. Rev. Lett. 116, 097204 (2016).

[20] R. Lebrun, A. Ross, S. A. Bender, A. Qaiumzadeh, L. Baldrati, J. Cramer, A. Brataas, R. A. Duine, and M. Kläui, "Tunable long-distance spin transport in a crystalline antiferromagnetic iron oxide," Nature 561, 222-225 (2018).

[21] Alexander Mook, Börge Göbel, Jürgen Henk, and Ingrid Mertig, "Magnon transport in noncollinear spin textures: Anisotropies and topological magnon Hall effects," Phys. Rev. B 95, 020401 (2017).

[22] Benedetta Flebus, Yaroslav Tserkovnyak, and Gregory A. Fiete, "Interfacial spin Seebeck effect in noncollinear magnetic systems," Phys. Rev. B 99, 224410 (2019).

[23] Bo Li, Shane Sandhoefner, and Alexey A. Kovalev, "Intrinsic spin Nernst effect of magnons in a noncollinear antiferromagnet," Phys. Rev. Research 2, 013079 (2020). 
[24] We implicitly assume that the usual Zeeman term is the only field-dependent term in the spin Hamiltonian.

[25] T. Holstein and H. Primakoff, "Field dependence of the intrinsic domain magnetization of a ferromagnet," Phys. Rev. 58, 10981113 (1940).

[26] The Supplemental Material contains a detailed derivation of linear spin-wave theory (Sec. I) and of the spin, orbital, and full magnetization (Sec. II-V). Extra information on the orderby-disorder study in kagome antiferromagnets and temperature dependence of the in-plane magnetization components is provided (Sec. VI). Moreover, we outline the linear spin-wave theory for pyrochlore ferromagnets and the basics of the transport calculations (Sec. VII). Finally, we address the magnonic orbital moment both in chiral (Sec. VIII) and dipolar ferromagnets (Sec. IX). Additional reference to Refs. $47-51$ is provided. URL: [inserted by publisher].

[27] Field-dependent terms in the spin Hamiltonian beyond the Zeeman term, e. g. ring exchange [46], yield additional contributions. For a general definition and more details, see SM [26].

[28] I. Dzyaloshinsky, "A thermodynamic theory of "weak" ferromagnetism of antiferromagnetics," J. Phys. Chem. Sol. 4, 241 (1958).

[29] Tôru Moriya, "Anisotropic superexchange interaction and weak ferromagnetism," Phys. Rev. 120, 91-98 (1960).

[30] M. Elhajal, B. Canals, and C. Lacroix, "Symmetry breaking due to Dzyaloshinsky-Moriya interactions in the kagomé lattice," Phys. Rev. B 66, 014422 (2002).

[31] Alexander Mook, Jürgen Henk, and Ingrid Mertig, "Thermal Hall effect in noncollinear coplanar insulating antiferromagnets," Phys. Rev. B 99, 014427 (2019).

[32] For parameters of $\mathrm{CdCu}_{3}(\mathrm{OH})_{6}\left(\mathrm{NO}_{3}\right)_{2} \cdot \mathrm{H}_{2} \mathrm{O}$-we used $S=1 / 2$, $g=2.1, D_{\|}=D_{z}=0.1 J$, and $J=3.87 \mathrm{meV}$ and $\phi=\pi / 2$-we find that the weak moment accounts for $\lesssim 1 \%$ of the experimentally found $7.93 \times 10^{-2} \mu_{\mathrm{B}} / \mathrm{Cu}$ [52]. Similar estimates may apply to Ca-kapellasite [53] and $\mathrm{YCu}_{3}(\mathrm{OH})_{6} \mathrm{Cl}_{3}$ [54].

[33] Y. Onose, T. Ideue, H. Katsura, Y. Shiomi, N. Nagaosa, and Y. Tokura, "Observation of the magnon Hall effect," Science 329, 297-299 (2010).

[34] Maged Elhajal, Benjamin Canals, Raimon Sunyer, and Claudine Lacroix, "Ordering in the pyrochlore antiferromagnet due to Dzyaloshinsky-Moriya interactions," Phys. Rev. B 71, 094420 (2005).

[35] This field models an anisotropy; see SM [26, Sec. VII] for details.

[36] Kira Riedl, Daniel Guterding, Harald O. Jeschke, Michel J. P. Gingras, and Roser Valentí, "Ab initio determination of spin hamiltonians with anisotropic exchange interactions: The case of the pyrochlore ferromagnet $\operatorname{lu}_{2} \mathrm{~V}_{2} \mathrm{O}_{7}$," Phys. Rev. B 94, 014410 (2016).

[37] Jakub Železný, Yang Zhang, Claudia Felser, and Binghai Yan, "Spin-polarized current in noncollinear antiferromagnets," Phys. Rev. Lett. 119, 187204 (2017).

[38] Hosho Katsura, Naoto Nagaosa, and Patrick A. Lee, "Theory of the thermal Hall effect in quantum magnets," Phys. Rev. Lett. 104, 066403 (2010).

[39] Ryo Matsumoto and Shuichi Murakami, "Rotational motion of magnons and the thermal Hall effect," Phys. Rev. B 84, 184406
(2011).

[40] Ryo Matsumoto and Shuichi Murakami, "Theoretical prediction of a rotating magnon wave packet in ferromagnets," Phys. Rev. Lett. 106, 197202 (2011).

[41] Ryo Matsumoto, Ryuichi Shindou, and Shuichi Murakami, "Thermal Hall effect of magnons in magnets with dipolar interaction,” Phys. Rev. B 89, 054420 (2014).

[42] Alexander Mook, Jürgen Henk, and Ingrid Mertig, "Magnon Hall effect and topology in kagome lattices: A theoretical investigation," Phys. Rev. B 89, 134409 (2014).

[43] S. Seki, X. Z. Yu, S. Ishiwata, and Y. Tokura, "Observation of skyrmions in a multiferroic material," Science 336, 198-201 (2012).

[44] Aurélien Manchon, Papa Birame Ndiaye, Jung-Hwan Moon, Hyun-Woo Lee, and Kyung-Jin Lee, "Magnon-mediated Dzyaloshinskii-Moriya torque in homogeneous ferromagnets," Phys. Rev. B 90, 224403 (2014).

[45] Jairo Sinova, Sergio O. Valenzuela, J. Wunderlich, C. H. Back, and T. Jungwirth, "Spin Hall effects," Rev. Mod. Phys. 87, 12131260 (2015).

[46] Li chuan Zhang, Fabian R. Lux, Jan-Philipp Hanke, Patrick M. Buhl, Sergii Grytsiuk, Stefan Blgel, and Yuriy Mokrousov, "Orbital Nernst effect of magnons," (2019), arXiv:1910.03317.

[47] S. Toth and B. Lake, "Linear spin wave theory for single-q incommensurate magnetic structures," Journal of Physics: Condensed Matter 27, 166002 (2015).

[48] A. L. Chernyshev and P. A. Maksimov, "Damped topological magnons in the kagome-lattice ferromagnets," Phys. Rev. Lett. 117, 187203 (2016).

[49] Na Su, Feiye Li, Yuanyuan Jiao, Ziyi Liu, Jianping Sun, Bosen Wang, Yu Sui, Haidong Zhou, Gang Chen, and Jinguang Cheng, "Asymmetric ferromagnetic criticality in pyrochlore ferromagnet $\mathrm{Lu}_{2} \mathrm{~V}_{2} \mathrm{O}_{7}$," Science Bulletin 64, 1222 - 1227 (2019).

[50] M. Mena, R. S. Perry, T. G. Perring, M. D. Le, S. Guerrero, M. Storni, D. T. Adroja, Ch. Rüegg, and D. F. McMorrow, "Spinwave spectrum of the quantum ferromagnet on the pyrochlore lattice $\mathrm{Lu}_{2} \mathrm{~V}_{2} \mathrm{O}_{7}$," Phys. Rev. Lett. 113, 047202 (2014).

[51] Benedetta Flebus, Ka Shen, Takashi Kikkawa, Ken-ichi Uchida, Zhiyong Qiu, Eiji Saitoh, Rembert A. Duine, and Gerrit E. W. Bauer, "Magnon-polaron transport in magnetic insulators," Phys. Rev. B 95, 144420 (2017).

[52] Ryutaro Okuma, Takeshi Yajima, Daisuke Nishio-Hamane, Tsuyoshi Okubo, and Zenji Hiroi, "Weak ferromagnetic order breaking the threefold rotational symmetry of the underlying kagome lattice in $\mathrm{CdCu}_{3}(\mathrm{OH})_{6}\left(\mathrm{NO}_{3}\right)_{2} \cdot \mathrm{H}_{2} \mathrm{O}$," Phys. Rev. B 95, 094427 (2017).

[53] Y. Ihara, H. Yoshida, K. Arashima, M. Hirata, and T. Sasaki, "Anisotropic magnetic excitations from single-chirality antiferromagnetic state in Ca-kapellasite," Phys. Rev. Research 2, 023269 (2020).

[54] A. Zorko, M. Pregelj, M. Gomilšek, M. Klanjšek, O. Zaharko, W. Sun, and J.-X. Mi, "Negative-vector-chirality $120^{\circ}$ spin structure in the defect- and distortion-free quantum kagome antiferromagnet $\mathrm{YCu}_{3}(\mathrm{OH})_{6} \mathrm{Cl}_{3}$," Phys. Rev. B 100, 144420 (2019). 